\title{
Joint optimization of link scheduling and resource allocation in cooperative vehicular networks
}

\author{
Qiang Zheng ${ }^{1}$, Kan Zheng ${ }^{1 *}$, Periklis Chatzimisios ${ }^{2}$ and Fei Liu ${ }^{3}$
}

\begin{abstract}
Vehicular networks are a cornerstone of the envisioned intelligent transportation system (ITS) by enabling vehicles to communicate with each other via vehicle-to-vehicle (V2V) communications to overcome the current and future needs for increasing traffic safety and efficiency. In this paper, we employ the knapsack problem (KP) to formulate the problem of cooperative scheduling and radio resource management in vehicular networks for non-real time services. For the sake of maximizing sum utility (MSU) of the networks, we propose two-dimensional-multi-choice knapsack problem (2D-MCKP)-based scheduling scheme to select the coordinator vehicles for the sink vehicle and allocate radio resource to V2V and vehicle-to-infrastructure (V2I) links to solve the MSU optimization problem. Simulation results indicate that the proposed scheme significantly improves the average utility and average data rates with sustainable computational complexity. Moreover, the designed cooperative communication system achieves higher spectral efficiency and better fairness.
\end{abstract}

Keywords: V2V communications; Cooperative communications; Cooperative vehicular networks; Two-dimensional-multi-choice knapsack problem; Resource allocation

\section{Introduction}

Vehicular networking serves as one of the most important enabling technologies in the envisioned intelligent transportation system (ITS) which mainly consists of two transmission categories, i.e., vehicle-to-vehicle $(\mathrm{V} 2 \mathrm{~V}) \mathrm{com}$ munications which enable vehicles to communicate with each other and vehicle-to-infrastructure (V2I) communications which capacitate vehicles to communicate with roadside unit [1]. Explosive growth in information technology has enabled scores of innovation applications in vehicular networks, such as real-time update of congestion, weather conditions report, multimedia services, and online games $[2,3]$. In the foreseeable future, more and more vehicles will be equipped with wireless radio devices capable of V2V and V2I communications to contribute to a safer and more efficient driving experience. To satisfy the requirements of the applications in vehicular networks,

\footnotetext{
*Correspondence: zkan@bupt.edu.cn

1 Wireless Signal Processing and Network (WSPN) Lab, Key Lab of Universal Wireless Communications, Ministry of Education, Beijing University of Posts \& Telecommunications, Xitucheng Road, 100876, Beijing, China

Full list of author information is available at the end of the article
}

intensive scholars and researchers have been constantly working for the advance of the V2I technologies. Meanwhile, extensive investigations and trials have been carried out to V2V communications resulting in the development of various standards, such as IEEE 802.11p [4] and the 1609 family of standards $[5,6]$.

Most of the innovation applications in vehicular networks such as mobile television, multimedia services, or security applications require high throughput and/or low delay. However, the rapid change of the topology of vehicular networks due to the mobility of the participating vehicles results in the degradation of the performance of vehicular communications and makes evident the need for more robust protocols or schemes to improve the system capacity. To eliminate the negative impact of the wireless channel fading and exploit spatial diversity, significant amount of research work has been carried out on the areas of cooperative communications and resource scheduling in cooperative vehicular networks [7-9]. The core idea of cooperative communications in vehicular networks is that when the channel between the base station (BS) and the destination is unreliable, another vehicle that encounters

\section{Springer}

(C) 2015 Zheng et al. This is an Open Access article distributed under the terms of the Creative Commons Attribution License (http://creativecommons.org/licenses/by/4.0), which permits unrestricted use, distribution, and reproduction in any medium, provided the original work is properly credited. 
much better channel conditions than the BS is selected to forward the packets to the destination vehicle (DV) and, thus, a significant gain for the whole system is achieved. In [10], authors develop and analyze low-complexity cooperative protocol that combats fading caused by multipath propagation in wireless networks. Moreover, amplify-andforward (AF) and decode-and-forward (DF) have been already developed to enhance the spectral diversity to reduce the outage probability. In [1], the authors design a new distributed cooperative relay medium access control (MAC) protocol to maximize the vehicles' achieved system throughput and service distance by adaptively selecting among suitable transmission modes and relay nodes according the channel quality. Simulation results show that the Markov-chain-based modeling outperforms existing schemes under the same networks scenarios and maximize the achieved system throughput and service distance. Cross-layer approaches for cooperative diversity networks are also investigated, combining the cooperative diversity concept with joint optimization of the physical and MAC layers $[9,11]$. The network coding (NC) technique is employed to improve the performance of vehicular communications. In [12], the authors investigate the information spread problem in a joint V2I and V2V communication system, and network-coding-based technique is used to mitigated the interference caused by relaying signal. However, little research effort has been devoted to resource allocation and relay selection for cooperative vehicular communications. A downlink resource allocation scheme for V2V2I communication system is proposed in [13]. Both an infrastructure and a vehicle can form multiple direction beams via smart antenna in order to transmit multiple data streams simultaneously. But the authors in [13] only consider the resource allocation on V2V link and V2I links and how to avoid the co-channel interference between V2I and V2V links with no regard to relay selection. In $[14,15]$, several relay selection and resource allocation for cooperative communication are presented. The main idea of them is that V2V communication is used to complement V2I communication. A bipartite graph-based scheduling scheme to allocate the V2I and V2V links for both 1-hop and 2-hop communications is proposed in [14]. The Kuhn-Munkres algorithm is applied to solve the problem of maximum weighted matching of the constructed bipartite graph of the vehicular networks, and simulations indicate that the proposed solution performs extremely close to the optimal one and achieves better fairness. But the radio resource are allocated equally to every link, which is not the optimal. In [16], authors take the resource allocation of V2V links into consideration when making relay selection decision. A cooperative social network and its dynamic bandwidth allocation algorithm are proposed in [17]. In [17], the authors pay more attention to upper layer social networks and the proposed scheme is divided into two step: relay selection and bandwidth allocation. However, the vehicles select the closest relay station (RS) to forward data, which is not the optimal selection and the link quality is not consideration. In addition, in the simulation, the interaction among vehicles is not taken into account. In [15], the long range (LR) transmission and short range (SR) transmission are proposed. The LR is based on Long-Term Evolution (LTE) and SR is based on IEEE 802.11p. The resource allocation in [15] only focuses on LR (LTE) links and the V2V links adopt multicast, which is suitable for delay-sensitive service, for example, public safety service. An enhanced connectivity scheme is proposed in [18], in which the author proposed resource allocation algorithm for joint operation of SR V2V communications and LR LTE communications in vehicular networks. Considerable research effort that has been devoted to cooperative communication in vehicular networks have proposed a number of resource allocation schemes or relay selection schemes, respectively. However, most of them have solved part of the problems appeared in cooperative vehicular networks and the proposed schemes are usually suboptimal. In our work, we propose the optimal scheduling scheme for cooperative vehicular communications, which takes relaying selection, resource allocation on V2I links and resource allocation on V2V links into consideration.

In our paper, we consider scenarios where a vehicle equipment (VE) can enjoy service through V2I connectivity through cellular communications, such as LTE. LTE is employed to support V2I communications for following reasons, i.e, high data rate, low latency, large coverage area, high energy efficient, robust interference-controlled, high penetration rate, and high-speed terminal support $[19,20]$. Nevertheless, several challenges lie ahead before LTE can be exploited in vehicular environments, e.g., in LTE, the VEs far away from the BS will suffer from much lower data rates due to poor radio links. To tackle this challenge, a cooperative relaying mechanism among neighboring vehicles is desired to be established for $\mathrm{V} 2 \mathrm{~V}$ communications through another OFDMA-based system [21]. Due to the limitation of V2V and V2I radio resources, the approach that we need to utilize in order to establish the V2V links and allocate the radio resources to V2I and $\mathrm{V} 2 \mathrm{~V}$ links is crucial to the performance of the vehicular network. We employ two-dimensional-multi-choice knapsack problem (2D-MCKP) to formulate the problem of scheduling and allocating resources to V2I and V2V links. The optimal solution is obtained through the 2DMCKP. Our approach actually enhances the total utility and achieves better fairness among VEs.

The main contributions of our work are summarized as follows:

- Formulation of the scheduling and allocating 
problem in the 2-hop vehicular network;

- Design of a optimal link scheduling and resources allocation scheme for vehicular networks with considerably lower computational complexity; and

- Performance comparison against the maximum sum rate (MSR) and BG-based schemes of [14].

The rest of our paper is organized as follows. The system model and problem formulation are illustrated in Section 2. Multidimensional-multi-choice knapsack problem-based (MD-MCKP) scheduling scheme is proposed in Section 3. Simulation scenarios and numerical results are presented and analyzed in Section 4. Finally, conclusions and future research are provided in Section 5.

\section{System model and problem formulation}

\subsection{System model}

As illustrated in Fig. 1, we consider the vehicular network with $N$ VEs, each of which has a single antenna and only one static roadside unit in the network [14]. The basic idea of cooperative relaying is that a vehicle encountering low data rates can be assisted for its downlink transmissions by the neighboring vehicles that encounter higher rates. These assisting vehicles are referred to as coordinator vehicles (CVs). With such assistance available, the roadside unit (RSU) will be able to transmit data utilizing a higher rates to the CVs which in turn will forward the data to the DV, which is termed as the sink vehicle (SV). Thus, a higher performance is achieved compared to the case that the low-rate vehicles were to transmit the data directly to the destination. Thus, the communication in the vehicular network is classified into two types, i.e., direct communication for CVs and cooperative communication for SVs. The main role of scheduling center is to collect the channel state information (CSI) of all vehicle users, determine which communication mode to be used and allocate the radio resource to V2V and V2I links according to collecting information. Before explaining the detail of operations, we provide in Table 1 a list of variables that will be used in this paper.

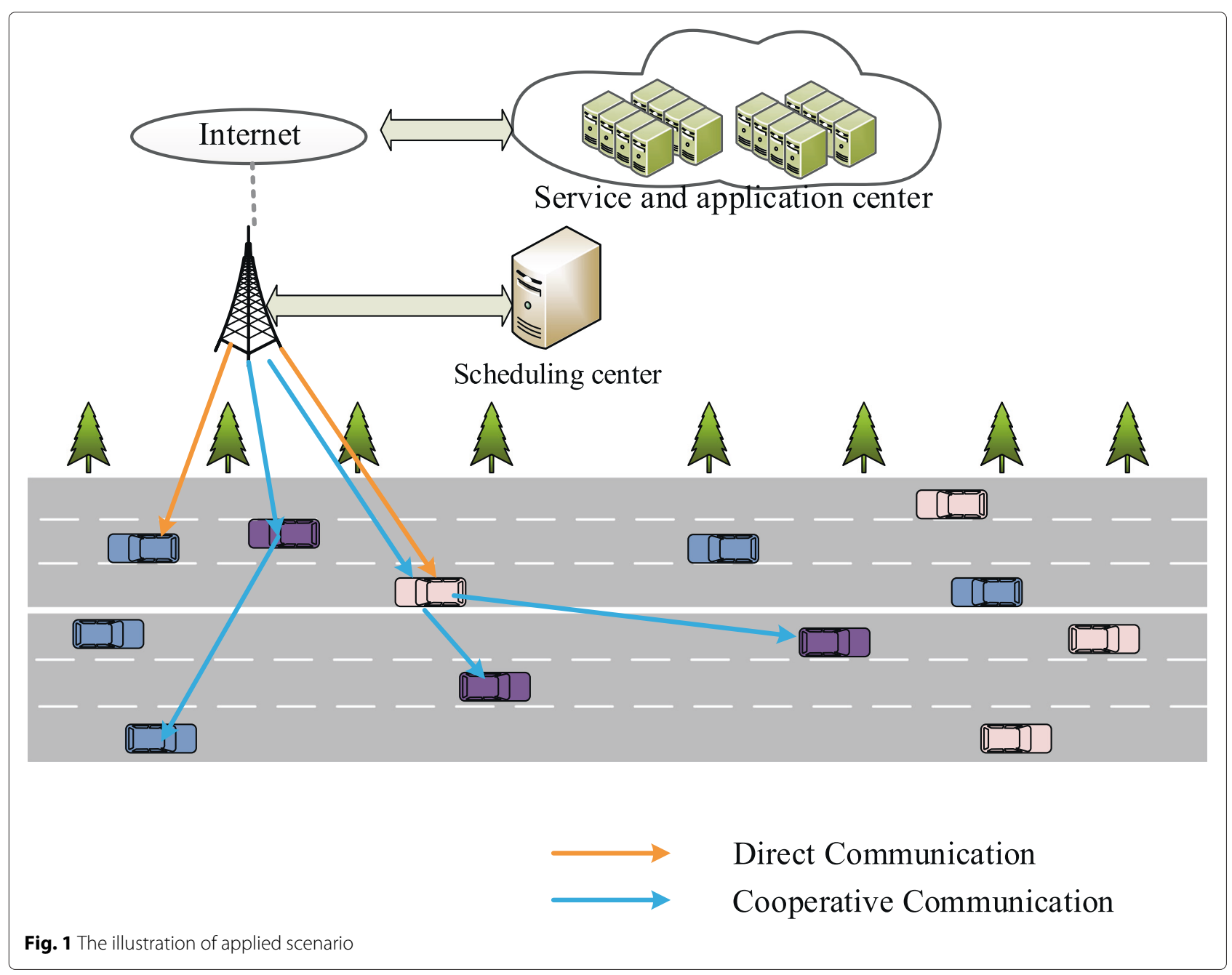


Table 1 Definitions for the notations

\begin{tabular}{ll}
\hline Symbol & Description \\
\hline$N$ & The total number of vehicles in the scenarios \\
$N_{B}$ & The number of vehicles that directly communicate with eNB \\
$N_{V}$ & The number of vehicles that cooperatively communicate with eNB \\
$K_{B}$ & The number of radio resources available for direct communication \\
$K_{V}$ & The number of radio resources available for cooperative communication \\
$P_{B}$ & Transmit power over per bandwidth \\
$P_{T}$ & Total transmit power of eNB \\
$P_{i, j}$ & Transmit power for V2V link between vehicles $i$ and $j$ \\
$\tilde{P}_{T}$ & Total transmit power of each vehicle \\
$N_{0}$ & Unilateral power spectral density of AWGN \\
$W$ & System bandwidth \\
$N_{C}$ & The number of cells \\
$\beta$ & Path loss attenuation factor \\
A & Binary matrix that represents the resource allocation between CVs \\
B & Binary matrix that represents the resource allocation between SVS \\
$\eta_{B, i}$ & Achievable data rate of the ith CV \\
$\eta_{i, j}$ & Achievable data rate of the link between vehicle $i$ and $j$ \\
$n_{i}$ & Radio resource allocated for the ith CV \\
$n_{i j}$ & Radio resource allocated for link between vehicle $i$ and $j$ \\
\hline
\end{tabular}

The overall operational processes can be concluded as follows,

- The vehicles and evolved NodeB (eNB) broadcast signaling to indicate the existing of themselves via control channel periodically;

- The vehicles which receive the signaling to estimate the V2V and V2I link quality and update the CSI;

- Then, the vehicles upload the CSI to the scheduling center;

- Based on CSI, the scheduling center schedules links, allocates radio resources and broadcasts the scheduling results via control channel.

\subsubsection{Direct communications}

Direct communication via a direct link between a VE and the eNB, i.e., the V2I link, is based on the LTEAdvanced specification. As specified in [22], the entire radio resources are divided into resource blocks (RBs) along the time and/or frequency domain and, thus, the VEs can share all the RBs through the given scheduling algorithm, assuming that $K_{B}$ RBs are available for the downlink transmission. According to the Shannon's theorem, the achievable data rate of the $i$ th $\mathrm{CV}$ is expressed by

$$
\begin{gathered}
\eta_{B, i}\left(n_{i}\right)=n_{i} W \log _{2}\left(1+\frac{\beta_{B, i} P_{B}}{\sum_{m=1, m \neq B}^{N_{C}} \beta_{m, i} P_{m}+N_{0} W}\right), \\
1 \leq i \leq N, 1 \leq n_{i} \leq K_{B},
\end{gathered}
$$

where $W$ is the basic bandwidth of one $\mathrm{RB}, n_{i}$ is the number of RBs allocated to $i$ th $\mathrm{CV}$ for direct communication by the scheduling center, $N_{C}$ is the number of cells occupying the same radio resources and only the inter-cell interference from the neighbor cells are taken into account, $\beta_{m, i}$ is the path loss attenuation factor from the eNB of cell $m$ to the $i$ th $\mathrm{CV}, P_{m}$ is the transmit power over $W$ bandwidth, i.e., $P_{m}=\frac{P_{T}}{K_{B}}, P_{T}$ is the total transmit power of the eNB, and $N_{0}$ is unilateral power spectral density of the additive white Gaussian noise (AWGN).

\subsubsection{Cooperative communication}

Cooperative communication, which involves V2I and $\mathrm{V} 2 \mathrm{~V}$ communications, is designed for the SVs. V2I communication is also based on LTE-Advanced system. However, the $\mathrm{V} 2 \mathrm{~V}$ communication is operating in out-of-band relaying mode using another orthogonal frequency-division multiple access (OFDMA)-based system. The specification of the OFDMA-based system is the same with the LTE-Advanced system but at a higher frequency band and low transmission power. For simplicity purposes, we provide the following assumptions:

- Each SV can be assisted by only one CV at any time;

- One CV can forward data to more than one SV;

- The DF relaying is applied at CV. 
When the $j$ th $\mathrm{SV}$ is assisted by the $i$ th $\mathrm{CV}$, the equivalent achievable data rate that the $j$ th SV can obtain is given by

$\tilde{\eta}_{B, i, j}\left(n_{i, j}, \tilde{n}_{i, j}\right)=\min \left\{\eta_{B, i}\left(n_{i, j}\right), \eta_{i, j}\left(\tilde{n}_{i, j}\right)\right\}, 1 \leq i, j \leq N, i \neq j$,

where $n_{i, j}$ is the number of RBs allocated to $i$ th CV for transmit the data of $j$ th $\mathrm{SV}$ to $\mathrm{CV} i$ from the eNB, $\tilde{n}_{i, j}$ is the number of RBs allocated to the $i$ th $\mathrm{CV}$ for its forwarding data to the $j$ th SV, i.e., $1 \leq n_{i, j} \leq K_{B}, 1 \leq \tilde{n}_{i, j} \leq K_{V}$, and $K_{V}$ is the total number of RBs for V2V communication. $\eta_{i, j}\left(\tilde{n}_{i, j}\right)$ are the data rates of V2V link between CV $i$ and SV $j$ utilizing $\tilde{n}_{i, j}$ RBs. $\eta_{i, j}\left(\tilde{n}_{i, j}\right)$ can be defined as follows:

$$
\begin{aligned}
\eta_{i, j}\left(\tilde{n}_{i, j}\right) & =\tilde{n}_{i, j} W \log _{2}\left(1+\frac{\tilde{n}_{i, j} \beta_{i, j} P_{i j}}{\tilde{n}_{i, j} N_{0} W}\right) \\
& =\tilde{n}_{i, j} W \log _{2}\left(1+\frac{\beta_{i, j} i_{i, j}}{N_{0} W}\right), 1 \leq i, j \leq N, i \neq j, 1 \leq \tilde{n}_{i, j} \leq K_{V},
\end{aligned}
$$

where $P_{i, j}$ is the transmit power on only one RB from $\mathrm{CV}$ $i$ to $\mathrm{SV} j$, i.e., $P_{i, j}=\tilde{P}_{T} / K_{V}, \tilde{P}_{T}$ is the total transmit power over all bandwidth of $\mathrm{V} 2 \mathrm{~V}$ links.

\subsection{Problem formulation}

Based on the network topology, we denote that $N_{B}$ and $N_{V}$ as the number of the CV and SV. Let the binary matrix $\mathbf{A}=\left\{a_{i, p} \mid a_{i, p} \in\{0,1\}\right\}_{N_{B} \times K_{B}}$ represent the resource allocation between CVs, where $a_{i, p}=1$ denotes that $p$ RBs are allocated to CV $i$ for direct communication, and $a_{i, p}=0$ otherwise. The establishment of V2V links and resource allocation on CVs and SVs are indicated by another binary matrix $\mathbf{B}=\left\{a_{i, j, p, q} \mid a_{i, j, p, q} \in\{0,1\}\right\}_{N_{B} \times N_{V} \times K_{B} \times K_{V}}$, where $a_{i, j, p, q}=1$ means that SV $j$ spends $q$ RBs in V2V link and $p$ RBs in V2I link assisted by CV $i$. Our goal is to find $A$ and B such that the objective functions are optimal with the limited bandwidth. Many resource management methods or relay-selecting mechanisms aim at maximizing throughput or spectral efficiency. However, the satisfaction of the user is not only linearly dependent on plain quality of service (QoS). Utility theory provides the reasonable methods to formulate the relationship between user experience and various QoS metrics. A utility function is used as an effective trade-off between spectral efficiency and fairness of resource allocation. The utility function maps the performance criteria into a real number used as a metric to quantify the satisfaction [23]. From the aspect of the user, the most important factor affecting users' satisfaction is the data rate of communication. Therefore, in this paper, the utility is assumed to be the function of data rate.
The utility-based resource allocation scheme that aims at maximizing the total utility is defined as

$$
\begin{aligned}
\max \left\{\sum_{i=1}^{N_{B}}\right. & \left.\sum_{p=1}^{K_{B}} a_{i, p} f\left(\eta_{B, i}(p)\right)+\sum_{i=1}^{N_{B}} \sum_{j=1}^{N_{V}} \sum_{p=1}^{K_{B}} \sum_{q=1}^{K_{V}} b_{i, j, p, q} f\left(\tilde{\eta}_{B, i, j}(p, q)\right)\right\} \\
\text { s.t. } & \sum_{p=1}^{K_{B}} a_{i, p} \leq 1,1 \leq i \leq N_{B}, \\
& \sum_{i=1}^{N_{B}} \sum_{p=1}^{K_{B}} \sum_{q=1}^{K_{V}} b_{i, j, p, q} \leq 1,1 \leq j \leq N_{V}, \\
& \sum_{i=1}^{N_{B}} \sum_{p=1}^{K_{B}} a_{i, p} p+\sum_{i=1}^{N_{B}} \sum_{j=1}^{N_{V}} \sum_{p=1}^{K_{B}} \sum_{q=1}^{K_{V}} b_{i, j, p, q} p \leq K_{B}, \\
& \sum_{i=1}^{N_{B}} \sum_{j=1}^{N_{V}} \sum_{p=1}^{K_{B}} \sum_{q=1}^{K_{V}} b_{i, j, p, q} q \leq K_{V},
\end{aligned}
$$

where $f(\bullet)$ is the utility function of data rate. The first line in (4) is the objective function. The second and third lines in (4) account for the fact that each vehicle only has one solution to communicate with the eNB. The fourth line in (4) states the limited RBs number for V2I links, and the last line corresponds to the restrictions of RBs number used for V2V communications.

The utility function is modeled as a concave utility function based on the non-real-time services such as file transfer. This kind of service is not sensitive to delay and jitter but greatly demands on data rate. The utility function is fitted based on non-real-time services and can be described mathematically as follows,

$$
f(x)=\frac{\log _{2}\left(a \frac{x}{R_{\max }}+b\right)}{\log _{2}(a+b)},
$$

where $x$ is the achievable data rate, $R_{\max }$ is the maximum data rate, and the parameters $a$ and $b$ are used for the normalization of the function [23]. The utility function is adopted for two purposes: 1) to ensure the fairness among vehicle users and 2) to represent the fact that the user satisfaction is not increasing linearly with the data rate.

\section{Multidimensional-multi-choice knapsack problem-based scheduling scheme}

In this section, we propose a link scheduling-scheme based on MD-MCKP to seek the optimal solution to establish the $\mathrm{V} 2 \mathrm{~V}$ links and to distribute radio resource units to V2I and V2V links for downlink transmissions. The vehicles with poor V2I links are chosen as SVs which communicate with eNB involving both V2I and V2V links. The other vehicles with better V2I links can act as CVs to forward data to SVs. We first give a brief introduction to MD-MCKP problem, followed by the proposal of the MD-MCKP-based scheduling scheme. 


\subsection{MD-MCKP problem}

The MD-MCKP problem is a new proposed algorithm, which integrates the 0-1 multidimensional knapsack problem (0-1 MDKP) and MCKP [24]. 0-1 MDKP and MCKP are generalization of $0-1$ knapsack problem and special case of general 0-1 integer programming.

Formally, the 0-1 MDKP problem may be defined as follows:

$$
\begin{array}{ll}
\max & z=\sum_{j=1}^{n} p_{j} x_{j} \\
\text { s.t } & \sum_{j=1}^{n} w_{i}^{j} x_{j} \leq c_{i}, \quad i \in I=\{1, \ldots, m\}, \\
& x_{j} \in\{0,1\}, \quad j \in J=\{1, \ldots, n\} .
\end{array}
$$

where $n$ is a number of items and $m$ is a number of knapsack's constraints with capacities $c_{i}(i \in I)$, associated weights $w_{i}^{j}(i \in I ; j \in J)$ and the profits $p_{j}(j \in J)$. The objective is to find a feasible subset of the set items that yields a maximum profit. The matrix $W$ and the vectors $\mathbf{c}$ and $\mathbf{p}$ consist in real-valued constants that satisfy $W \geq 0, c \geq 0$, and $p \geq 0$. For simplicity, we only give a description about 2DKP, which is defined as follows:

$$
\begin{array}{ll}
\max & z=\sum_{j=1}^{n} p_{j} x_{j} \\
\text { s.t } & \sum_{j=1}^{n} w_{i}^{j} x_{j} \leq c_{i}, \quad i \in I=\{1,2\}, \\
& x_{j} \in\{0,1\}, \quad j \in J=\{1, \ldots, n\} .
\end{array}
$$

The 2DKP cannot be solved in a time bounded by a polynomial in $n$. However, admit a pseudo-polynomial algorithm, i.e., an algorithm whose time complexity is bounded by a polynomial in $n$ and $c$. In fact, it can easily be verified that the following dynamic programming recursions solve the 2DKP [24].

Given any one instance of the knapsack problem, consider the sub-instance defined by items $1, \ldots, j$ and capacity $u$ and $v\left(j \leq n, u \leq c_{1}, v \leq c_{2}\right)$. Let $f_{j}(u, v)$ be the corresponding optimal solution value $\left(f_{j}(u, v)=-\infty\right.$, if no feasible solution exists). The optimal solution value of the problem $f_{n}\left(c_{1}, c_{2}\right)$ can thus be obtained by iteratively applying the following recursive formulae:

$$
\begin{aligned}
& f_{1}(u, v)=\left\{\begin{array}{cc}
p_{1} \text { for } w_{1}^{1} \leq u \leq c_{1}, w_{2}^{1} \leq v \leq c_{2}, \\
0 & \text { otherwise, }
\end{array}\right. \\
& f_{j}(u, v)=\max \left(f_{j-1}(u, v), f_{j-1}\left(u-w_{1}^{j}, v-w_{2}^{j}\right)\right), \\
& \text { for } j=2, \ldots, n \text { and } u=0, \ldots, c_{1}, v=0, \ldots, c_{2} .
\end{aligned}
$$

The multiple-choice knapsack problem (MCKP), also known as the knapsack problem (KP) with generalized upper bound (GNU) constraints, is a 0-1 knapsack problem in which a partition $N_{1} \ldots N_{r}$ of the item set $N$ is given. It is required that exactly one item per subset is selected. Formally,

$$
\max z=\sum_{j=1}^{n} p_{j} x_{j}
$$

subject to

$$
\begin{aligned}
& \sum_{j=1}^{n} w_{j} x_{j} \leq c, \\
& \sum_{j \in N_{k}}^{n} x_{j}=1, k=1, \ldots, r, \\
& x_{j}=0 \text { or } 1, j \in N=\{1, \ldots, n\}=\bigcup_{k=1}^{r} N_{k}, \\
& N_{i} \cap N_{j}=\emptyset, i \neq j .
\end{aligned}
$$

The problem is NP-hard, since any instance of KP, having $r$ elements of profit $p_{j}$ and weight $w_{j}(j=1, \ldots, r)$ and capacity $c$, is equivalent to the instance of MCKP obtained by setting $n=2 r, p_{j}=w_{j}=0$ for $j=r+1, \ldots, 2 r$ and $N_{k}=\{k, r+k\}$ for $k=1, \ldots, r$. MCKP can be solved in pseudo-polynomial time through dynamic programming as follows. Given a pair of integers $l(1 \leq l \leq r)$ and $\tilde{c}$ $(0 \leq \tilde{c} \leq c)$, consider the sub-instance of MCKP consisting of subset $N_{1}, \ldots, N_{l}$ and capacity $\tilde{c}$. Let $f_{l}(\tilde{c})$ denote its optimal solution value, i.e.,

$$
\begin{aligned}
& f_{l}(\tilde{c})=\max \left\{\sum_{j \in \tilde{N}} p_{j} x_{j}: \sum_{j \in \tilde{N}} w_{j} x_{j} \leq \tilde{c}, \sum_{j \in N_{k}} x_{j}=1,\right. \\
& \left.\mathrm{k}=1, \ldots, l, x_{j}=0 \text { or } 1, \text { for } \mathrm{j} \in \tilde{\mathrm{N}}\right\},
\end{aligned}
$$

where $\tilde{N}=\bigcup_{k=1}^{l} N_{k}$, and assume that $f_{l}(\tilde{c})=-\infty$ if the sub-instance has no feasible solution. Let

$$
\bar{w}_{k}=\min \left\{w_{j}: j \in N_{k}\right\} \quad \text { for } \mathrm{k}=1, \ldots, r,
$$

clearly,

$f_{1}(\tilde{c})=\left\{\begin{array}{cl}-\infty & \text { for } \tilde{c}=0, \ldots, \bar{w}_{1}-1, \\ \max \left\{p_{j}: j \in N_{1}, w_{j} \leq \tilde{c}\right\} & \text { for } \tilde{c}=\bar{w}_{1}, \ldots, c\end{array}\right.$

for $l=2, \ldots, r$, we then have

$f_{l}(\tilde{c})=\left\{\begin{array}{cl}-\infty & \text { for } \tilde{c}=0, \ldots, \sum_{k=1}^{l} \bar{w}_{k}-1, \\ \max \left\{f_{l-1}\left(\tilde{c}-w_{j}\right)+p_{j}: j \in N_{l}, w_{j} \leq \tilde{c}\right\} & \text { for } \tilde{c}=\sum_{k=1}^{l} \bar{w}_{k}, \ldots, c .\end{array}\right.$

The time complexity of $2 \mathrm{DKP}$ is $\mathrm{O}\left(n c_{1} c_{2}\right)$. 
The optimal solution is the state corresponding to $f_{r}(c)$. If we have $\sum_{k=1}^{r} \bar{w}_{k}>c$, the instance has no feasible solution, and we obtain $f_{r}(c)=-\infty$. For each $l$, the above computation requires $O\left(\left|N_{l}\right| c\right)$ operations, so the overall time complexity of the method is $O(n c)$.

Our proposed 2D-MCKP algorithm is defined as follows:

$$
\begin{array}{ll}
\max & z=\sum_{j=1}^{n} p_{j} x_{j}, \\
\text { s.t } & \sum_{j=1}^{n} w_{i}^{j} x_{j} \leq c_{i}, \quad i \in I=\{1,2\}, \\
& \sum_{j \in N_{k}}^{n} x_{j}=1, \quad k=1, \ldots, r, \\
& x_{j}=0 \text { or } 1, \quad j \in N=\{1, \ldots n\}=\bigcup_{k=1}^{r} N_{k}, \\
& N_{i} \cap N_{j}=\emptyset, \quad i \neq j .
\end{array}
$$

Note that $N$ indicates both the set of vehicles and its cardinality and the optimal solution value of the problem $f_{r}\left(c_{1}, c_{2}\right)$ can be achieved by iteratively applying the following recursive formulae:

$\overline{w_{k}^{i}}=\min \left\{w_{j}^{i}: j \in N_{k}\right\} \quad$ for $k=1, \ldots, r$, and $i=1,2$,

$f_{1}\left(\tilde{c}_{1}, \tilde{c}_{2}\right)=$
$\left\{\begin{array}{cc}\max \left\{p_{j}: j \in N_{1}, w_{j}^{1} \leq \tilde{c}_{1}, w_{j}^{2} \leq \tilde{c}_{2}\right\} & \text { for } \tilde{c}_{1}=\overline{w_{1}^{1}}, \ldots, c_{1}, \tilde{c}_{2}=\overline{w_{1}^{2}}, \ldots, c_{2} \\ 0 & \text { otherwise. }\end{array}\right.$ for $l=2, \ldots, r$, we make iterative process as follow, for $\tilde{c}_{1}=\sum_{k=1}^{l} \bar{w}_{k}^{1}, \ldots, c_{1}, \tilde{c}_{2}=\sum_{k=1}^{l} \bar{w}_{k}^{2}, \ldots, c_{2}$,

$$
\begin{array}{r}
f_{l}\left(\tilde{c}_{1}, \tilde{c}_{2}\right)=\max \left\{f_{l-1}\left(\tilde{c}_{1}-w_{j}^{1}, \tilde{c}_{2}-w_{j}^{2}\right)\right. \\
+p_{j}: j \in N_{l}, w_{j}^{1} \leq \tilde{c}_{1}, w_{j}^{2} \leq \tilde{c}_{2},
\end{array}
$$

otherwise,

$$
f_{l}\left(\tilde{c}_{1}, \tilde{c}_{2}\right)=0
$$

After the iterative process, the optimal solution is the state corresponding to $f_{r}\left(c_{1}, c_{2}\right)$.

An example of the 2D-MCKP algorithm is illustrated in Fig. 2 by considering five subsets. The first three subsets have 4 elements and the last two subsets have 12 elements. Table 2 lists the profit, first weight and second weight of every element in all subsets. The capacity of the first and second weights is 8 and 5 , respectively. The numbers in Fig. 2 represent the optimal solution at the corresponding states. In Fig. 2, the five tables correspond to $L_{i}(p, q)$, for $i=1,2,3,4,5$. In addition, it follows that the first weight varies along the columns and the second weight varies along the rows. After the backtracking, the optimal solution is obtained. The optimal selection $(1,1,2,9,11)$ to the corresponding subset is achieved, and the maximum profit is 23 . It must also be noticed that the optimal selection is not the unique one.

\subsection{D-MCKP-based scheduling scheme}

In this subsection, we apply the 2D-MCKP method to calculate the binary matrix $\mathbf{A}$ and $\mathbf{B}$ through three steps as follow,

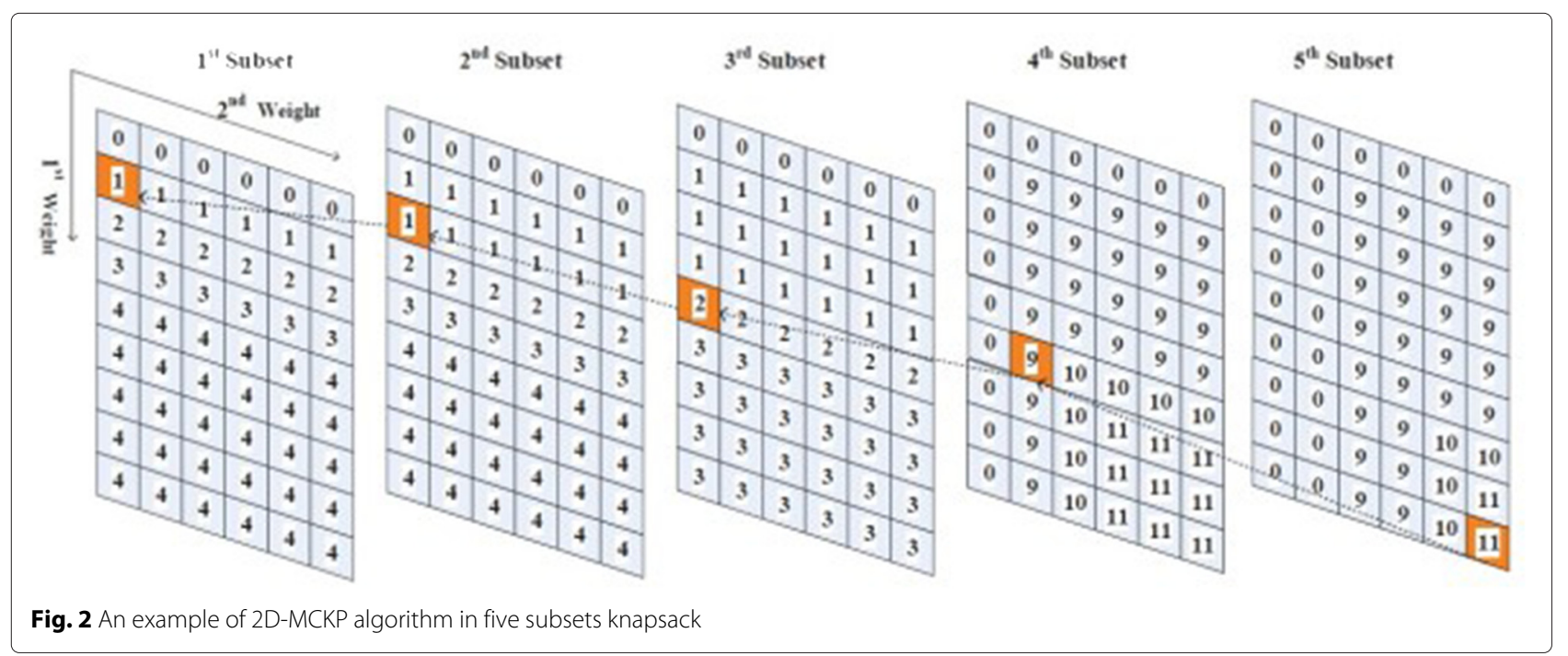


Table 2 The parameters of the 2D-MCKP example

\begin{tabular}{lccr}
\hline Subset & Profit & First weight & Second weight \\
\hline First subset & $(2,3,4,5)$ & $(1,2,3,4)$ & $(0,0,0,0)$ \\
Second subset & $(3,4,5,6)$ & $(1,2,3,4)$ & $(0,0,0,0)$ \\
Third subset & $(5,6,7,7)$ & $(1,2,3,4)$ & $(0,0,0,0)$ \\
Fourth subset & $(2,3,4,5,3,4,5,6,5,6,7,7)$ & $(1,2,3,4,1,2,3,4,1,2,3,4)$ & $(1,2,3,4,1,2,3,4,1,2,3,4)$ \\
Fifth subset & $(2,3,4,5,3,4,5,6,5,6,7,7)$ & $(1,2,3,4,1,2,3,4,1,2,3,4)$ & $(2,3,4,5,2,3,4,5,2,3,4,5)$ \\
\hline
\end{tabular}

1) Construct the group of 2D-MCKP.

For $\mathrm{VE} j$, group $G_{j}$ of selections is constructed. If vehicle $j$ is $C V$,

$$
G_{j}=\left\{g_{p}=\eta_{B, j}(p) \mid 1 \leq p \leq K_{B}\right\},
$$

otherwise,

$$
\begin{aligned}
& G_{j}=\left\{g_{\left.(i-1) K_{B} K_{V}+(q-1) K_{B}+p=\tilde{\eta}_{B, i, j}(p, q)\right\},}\right. \\
& \quad \text { for } 1 \leq p \leq K_{B}, 1 \leq q \leq K_{V}, 1 \leq i \leq N_{B}
\end{aligned} .
$$

where the set $G_{j}$ contains $K_{B}$ or $K_{B} \times K_{V} \times N_{B}$ elements, each of which represents the data rate corresponding to $\mathrm{VE} j$, i.e., $\eta_{B, j}(p)$ and $\tilde{\eta}_{B, i, j}(p, q)$ are both defined in Section 2.

Based on 2D-MCKP, we consider all the selections of $S V_{j}$ as a group and refer the $K_{B}$ and $K_{V}$ as the packet volumes. The mapping $C_{1}(\bullet)$ and $C_{2}(\bullet)$ is to obtain the first and second index of the solution. For CV $i$, the first dimension cost is $p$ and the second dimension cost is 0 , i.e., $C_{1}\left(g_{m}\right)=\bmod \left(m, K_{B}\right), C_{2}\left(g_{m}\right)=0$. However, for $\mathrm{SV} j$, the first dimension cost is $p$ and the second dimension cost is q, i.e., $C_{1}\left(g_{m}\right)=$ $\bmod \left(m, K_{B}\right), C_{2}\left(g_{m}\right)=\left\lceil\frac{\bmod \left(m, K_{B} K_{V}\right)}{K_{B}}\right\rceil$. The 2D-MCKP aims to achieve the maximum utility of all VEs, i.e.,

$$
\max \sum_{i=1 ; g_{m, i} \in G_{i}}^{N_{B}+N_{V}} f\left(g_{m, i}\right),
$$

subjected to

$$
\begin{gathered}
\sum_{i=1}^{N_{B}+N_{V}} C_{1}\left(g_{m, i}\right) \leq K_{B}, \\
\sum_{i=1}^{N_{B}+N_{V}} C_{2}\left(g_{m, i}\right) \leq K_{V} .
\end{gathered}
$$

Then the problem of resource management is converted to 2D-MCKP.

2) Solution of $2 \mathrm{D}-\mathrm{MCKP}$.

The solution of 2D-MCKP is described in detail in Algorithm 1.
Algorithm 1 The 2D-MCKP algorithm for solving the optimization problem

\section{Step 0: Initialization.}

Separate vehicles according to channel conditions of the V2I links.

Choose the worst $N_{V}$ vehicles as SVs according channel conditions.

$\bar{\eta}_{M U}^{*}(p, q)=\left\{\begin{array}{cc}0 & 0<p \leq K_{B}, 0<q \leq K_{V} \\ f(0) & \text { othersize, }\end{array}\right.$ the maximum sum of utility while the cost of

1-st dimension is $p$ and the cost of 2-nd dimension is q; $L_{j}(p, q)=0,0<p \leq K_{B}, 0<q \leq K_{V}$, the optimal number of RBs allocated to $\mathrm{VE} j$.

\section{Step 1: Construct groups.}

loop $j=1 \ldots N_{V}+N_{B}$

$G_{j}$ is constructed based on Equ. (27) and (28)

end loop

Step 2: Compare and label.

$$
\begin{aligned}
& \text { loop } j=1 \ldots N_{B}+N_{V} \text { for each VE } \\
& \text { loop } p=K_{B} \ldots 1 \\
& \text { loop } q=K V \ldots 1 \\
& \text { loop } g_{m} \in G_{j} \text { for every element in } G_{j} \\
& C=f\left(g_{m}\right), C_{1}=\bmod \left(m, K_{B}\right), \\
& C_{2}=C_{2}\left(g_{m}\right), \\
& \text { if } \bar{\eta}_{M U}^{*}(p, q)<\bar{\eta}_{M U}^{*}\left(p-C_{1}, q-C_{2}\right)+C, \\
& \text { then } \\
& \bar{\eta}_{M U}^{*}(p, q)=\bar{\eta}_{M U}^{*}\left(p-C_{1}, q-C_{2}\right)+C, \\
& L_{j}(p, q)=g_{m} \cdot \\
& \text { end if } \\
& \text { end loop } \\
& \text { end loop } \\
& \text { end loop } \\
& \text { end loop } \\
& \text { Step 3: Output and backtracking } \\
& \bar{\eta}_{M U}^{*}\left(K_{B}, K_{V}\right) \text { is the maximum sum utility of all } \\
& \text { VEs. } \\
& p=K_{B}, q=K_{V} \\
& \text { loop } j=N_{B}+N_{V} \ldots 1 \text { for each } S V \\
& L_{j}(p, q)=g_{m} \text { is the selected solution for VE } j, \\
& \text { set the value of corresponding } a_{i, p} \text { or } b_{i, j, p, q} \text { to } 1 \\
& p=p-C_{1}\left(L_{j}(p, q)\right), q=q-C_{2}\left(L_{j}(p, q)\right) \text {. } \\
& \text { end loop }
\end{aligned}
$$

\section{Step 3: Output and backtracking}


3) Optimization of $N_{V}$.

Based on the above discussion, we can calculate the total utility of all VEs, which is determined by parameter $N_{V}$ in a certain optimization problem. With a relatively large $N_{V}$, more SVs in poor conditions may be assisted by the CVs, and consequently increase the total utility. However, an overly large $N_{V}$ will limit the data rates of the $\mathrm{V} 2 \mathrm{~V}$ links, resulting in the reduction of the total utility. Furthermore, a too small $N_{V}$ is unable to make full use of the $\mathrm{V} 2 \mathrm{~V}$ resources, leading to waste of radio resources. In other words, the total utility is an unimodal function of $N_{V}$. The optimal $N_{V}$ can be obtained through binary search.

Through steps 1-3, the optimal V2V links and resource allocations are obtained. The SVs may have better SVs to be forwarded data, which results in that some CV has no SV to help.

\section{Performance evaluation}

In this section, we evaluate the performance of the proposed 2D-MCKP-based scheduling scheme via simulations. The experimental platform and considered simulation scenarios are implemented by utilizing the OPNET simulation software (version 14.5). Throughout our simulations, online one-way coupling is used, which eliminates the use of a sequential file-based process by having both simulators, traffic and communication, run in parallel [25].

\subsection{Simulation configuration}

The proposed scheduling scheme is applied in an intersection scenario as shown in Fig. 3b. Each direction of the intersection is $500 \mathrm{~m}$ in length and $18 \mathrm{~m}$ in width. It is assumed that there are five lanes in each direction of the intersection, three of which enter into the intersection and two leave out of it. In order to regulate the traffic flows, the traffic lights are added in the simulation. Table 3 lists the major parameters and configurations of the simulated network. According to the microscopic traffic model in [26], car-following and lane-changing model are employed in the simulation. The car-following theory is based on the assumption that the motion of a vehicle is governed exclusively by the motion of its preceding vehicle illustrated in Fig. 3a, the features of which are continuous in space, discrete in time and accident-free. The random lane-changing model is adopted in our simulations, and no overtaking model is used. The vehicles will randomly switch to one of all the possible lanes according to traffic laws.

V2I communications are based on the LTE-Advanced system. On the basis of [22], V2I communications data are transmitted via a $40-\mathrm{MHz}$ bandwidth at the $2-\mathrm{GHz}$ frequency point using $52 \mathrm{dBm}$ transmit power. Nevertheless, V2V communications use another OFDMA-based system
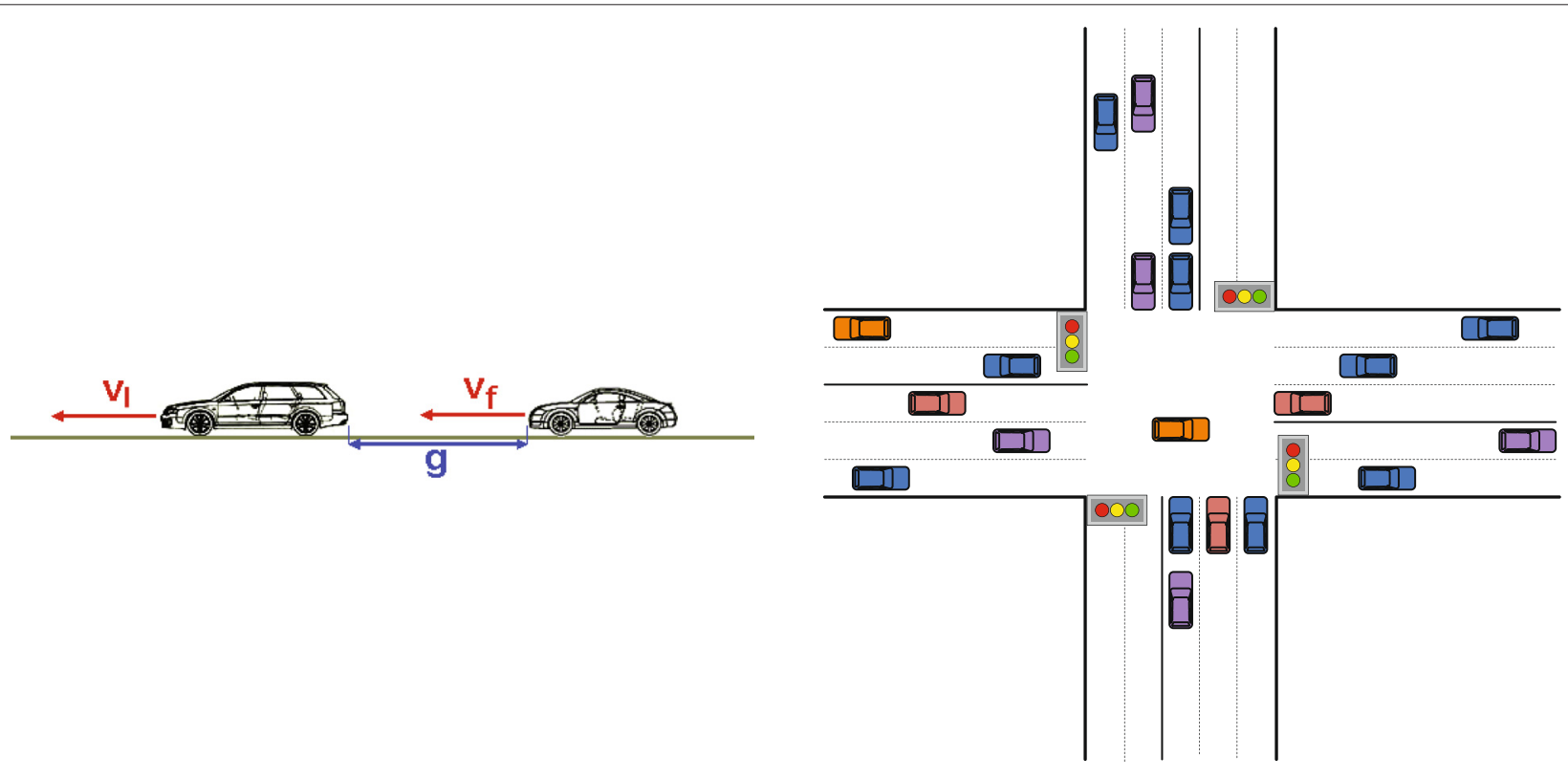

Fig. 3 The traffic simulation model: a car-following model and $\mathbf{b}$ simulation scenario 
Table 3 Simulation parameters

\begin{tabular}{|c|c|}
\hline Parameter & Value \\
\hline Cell radius & $500 \mathrm{~m}$ \\
\hline VE number & 10 to 100 \\
\hline Traffic model & Microscopic model in [26] \\
\hline Max drive speed & $126 \mathrm{~km} / \mathrm{h}(35 \mathrm{~m} / \mathrm{s})$ \\
\hline Acceleration & $2.6 \mathrm{~m} / \mathrm{s}^{2}$ \\
\hline Deceleration & $-4.5 \mathrm{~m} / \mathrm{s}^{2}$ \\
\hline Length of time slot & $1 \mathrm{~ms}$ \\
\hline Link scheduling interval & $1 \mathrm{~s}$ \\
\hline \multicolumn{2}{|l|}{ LTE configuration (V2I link) } \\
\hline Carrier frequency & $2 \mathrm{GHz}$ \\
\hline Bandwidth & $40 \mathrm{MHz}$ \\
\hline Transmit power of eNB & $52 \mathrm{dBm}$ for $40 \mathrm{MHz}$ \\
\hline \multicolumn{2}{|l|}{ Configuration of V2V link } \\
\hline Carrier frequency & $5.9 \mathrm{GHz}$ \\
\hline Bandwidth & $5 \mathrm{MHz}$ \\
\hline VE transmit power & $20 \mathrm{dBm}$ for $5 \mathrm{MHz}$ \\
\hline \multicolumn{2}{|c|}{ Utility function for elastic service } \\
\hline$a$ & 50 \\
\hline$b$ & 1 \\
\hline$R_{\max }$ & 6000 \\
\hline \multicolumn{2}{|l|}{ Path loss model } \\
\hline V2l link & $P(d)=1+37.6 \log _{10}\left(\frac{d}{1000}\right)$ \\
\hline V2V link & $\begin{aligned} l & =128.1-2 \mathrm{GHz} \\
P(d) & =P\left(d_{0}\right)+10 \gamma \log _{10}\left(\frac{d}{d_{0}}\right)+X_{\sigma}\end{aligned}$ \\
\hline
\end{tabular}

that supports three different bandwidths, i.e., 5,10 , and $20 \mathrm{MHz}$ operating in the 5.8- or 5.9-GHz frequency band. Four various power classes are defined in the standard, i.e., transmit power of $0,10,20$, and $28.8 \mathrm{dBm}$. In our simulations, $\mathrm{V} 2 \mathrm{~V}$ communications use $5-\mathrm{MHz}$ bandwidth at $5.8-\mathrm{GHz}$ frequency point with transmit power at 20
$\mathrm{dBm}$. Table 3 provides the path loss models of $\mathrm{V} 2 \mathrm{~V}$ and V2I links utilized in our simulations. The path loss model of V2I links is illustrated in detail in [22]. In case of V2V links, $P(d)$ is the received signal strength at a distance $d$, $\mathrm{P}\left(d_{0}\right)$ is the received signal strength at a reference distance $d_{0}, \gamma$ is the path loss exponent, and $\sigma$ is the standard deviation (STD) of the zero-mean Gaussian variable $X_{\sigma}$. In the simulation, $d_{0}=1, P\left(d_{0}\right)=43.9, \gamma=2.75$, and $\sigma=5.5$ [27].

\subsection{Results and analysis}

In this subsection, the MSR and the BG-based schemes proposed in [14] are both simulated for the purpose of performance comparison. The MSR and BG-based schemes are both subject to that one CV could forward data to at most one vehicle and the RBs are divided to all links equally.

Figure 4 illustrates the cumulative distribution functions (CDFs) of the data rates with the MSR, BG-based, and 2DMCKP schemes for the cases of $N=20, N=30$, and $N=$ 40 , respectively. The figure illustrates that the $2 \mathrm{D}$-MCKP scheme has a considerable improvement comparing to the MSR and BG-based schemes. Thus, the proposed scheme effectively reduces the number of vehicles at low data rate. For example, the CDF curves of 2D-MCKP achieve a much lower data rate level than other schemes, which means that we have less vehicles suffering from low data rate transmission.

In order to better demonstrate the utility performance, Fig. 5 shows the utility CDF and average vehicle utility of different radio resource allocation schemes. From the view of the utility efficiency, the average utility have obvious improvement compared with the MSR and BG-based

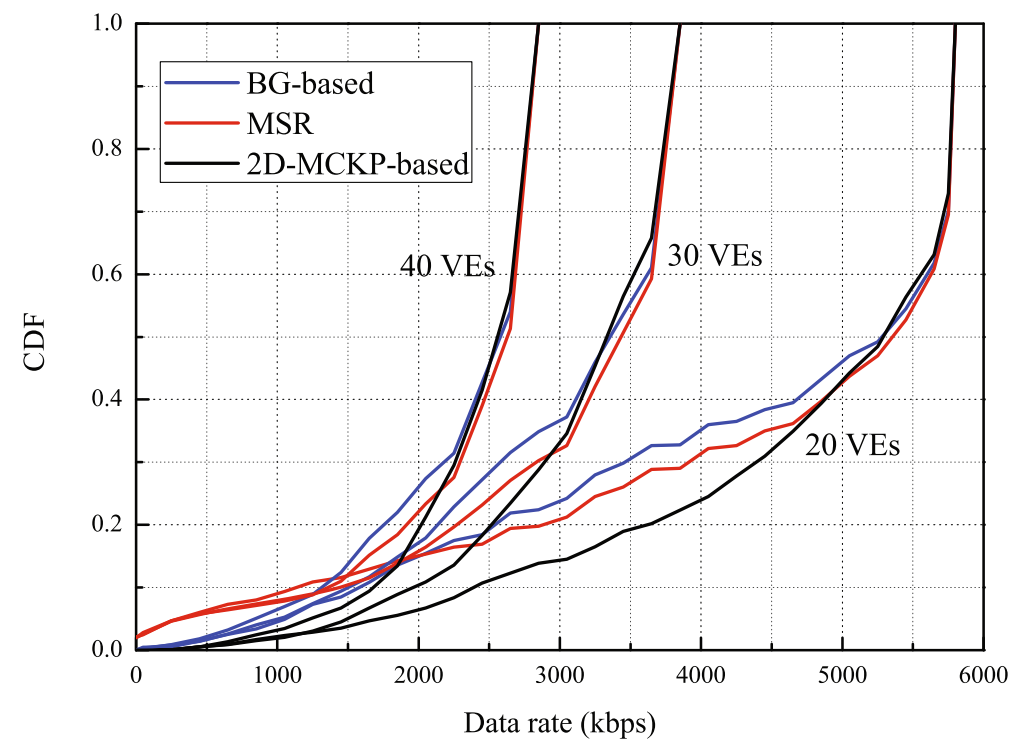

Fig. 4 CDFs of data rate with the MSR, BG-based, and 2D-MCKP schemes under various numbers of vehicles 


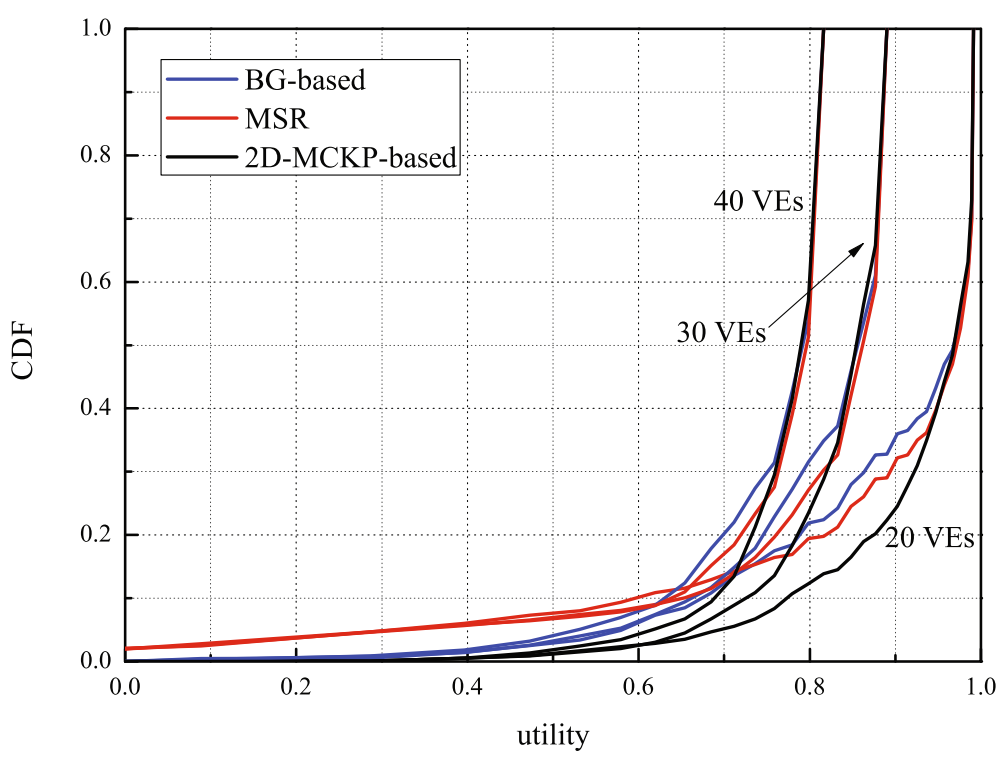

Fig. 5 CDFs of utility with the MSR, BG-based, and 2D-MCKP schemes under various numbers of vehicles

schemes, because the proposed 2D-MCKP scheme can allocate the RBs more reasonably to enhance the utility of total vehicular networks. From Figs. 4 and 5, we conclude that the proposed scheme not only significantly improves the total throughput but also increases the total utility of the vehicular networks.

Furthermore, in order to evaluate the performance of the different schemes in more detail, Table 4 provides the average data rate and data rate at $5 \% \mathrm{CDF}$ under different schemes, respectively. Compared against the MSR scheme and BG-based scheme, there is obvious performance improvement of the average data rates, and a significant enhancement on the data rates at the $5 \% \mathrm{CDF}$. This is due to the fact that our proposed scheme takes into account both links selection and resource allocation. Moreover, the computational complexity of the 2D-MCKP scheme is much more practical.
Figure 6 illustrates the average utility and standard deviation (STD) of utility with different schemes for the cases of $N=20, N=30$, and $N=40$. As shown in the top subfigure in Fig. 6, our proposed scheme outperforms the other two schemes on the performance metric of average utility. On the other hand, the average utility is reducing with the increasing of $N$, which is due to the fact that the total radio resource is fixed and the average radio resources allocated to each VE is reducing with the increasing of $N$. To better estimate the proposed scheme, STD of utility is introduced to demonstrate the fairness of schemes. As shown in Fig. 6, a lower STD can be achieved compared to other schemes, which actually leads to improve fairness. In conclusion, the 2D-MCKP scheme not only improves the average utility but guarantees the performance of vehicles in poor conditions.

Table 4 The average data rates and data rates at $5 \%$ CDF among different schemes

\begin{tabular}{|c|c|c|c|c|c|}
\hline \multirow[t]{2}{*}{ Number of VEs } & \multirow{2}{*}{ Scheduling scheme } & \multicolumn{2}{|c|}{$5 \%$ CDF } & \multicolumn{2}{|c|}{ Average data rate } \\
\hline & & Data rate (kbps) & Gain (\%) & Data rate (kbps) & Gain (\%) \\
\hline & MSR scheme & 407 & 100 & 4386 & 100 \\
\hline \multirow[t]{3}{*}{20} & BG-Based scheme & 1120 & 275.18 & 4350 & 99.18 \\
\hline & 2D-MCKP-based scheme & 1748 & 429.48 & 4552 & 103.79 \\
\hline & MSR scheme & 348 & 100 & 3014 & 100 \\
\hline \multirow[t]{3}{*}{30} & BG-Based scheme & 996 & 286.21 & 2990 & 99.20 \\
\hline & 2D-MCKP-based scheme & 1563 & 449.14 & 3110 & 103.19 \\
\hline & MSR scheme & 296 & 100 & 2336 & 100 \\
\hline \multirow[t]{2}{*}{40} & BG-Based scheme & 862 & 291.22 & 2320 & 99.32 \\
\hline & 2D-MCKP-based scheme & 1266 & 427.70 & 2430 & 104.02 \\
\hline
\end{tabular}




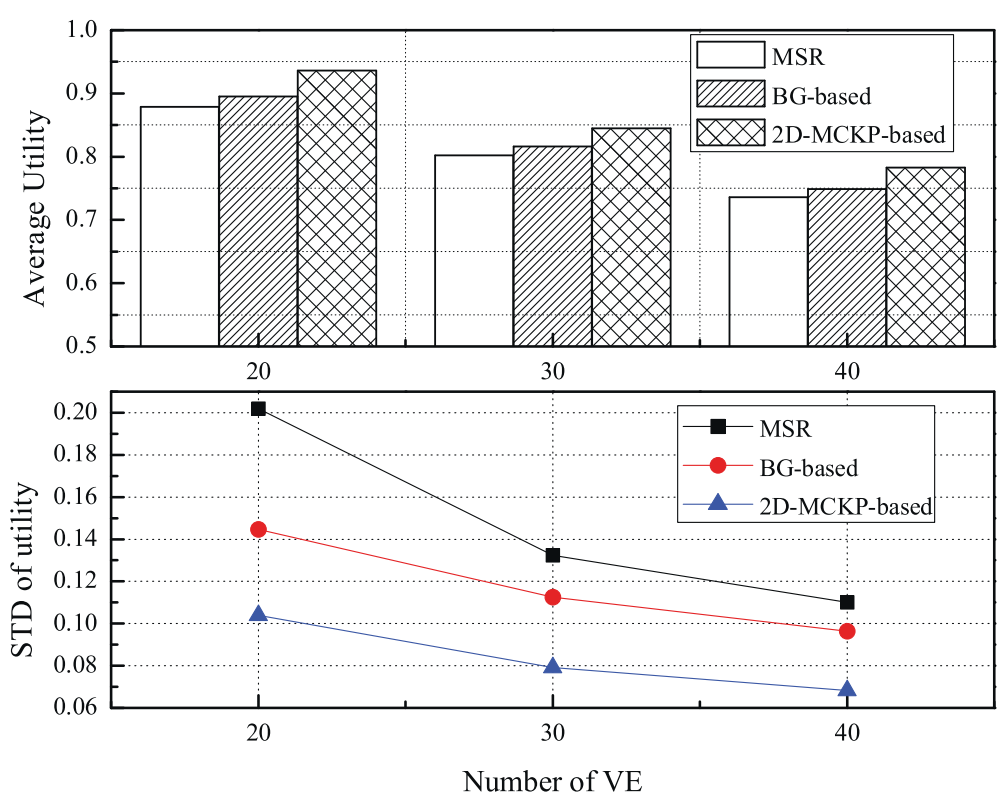

Fig. 6 Gain and STD of utility with different schemes with elastic service

We compute the spectral efficiency (SE) of BG-based, 2D-MCKP-based and non-cooperative schemes as shown in Fig. 7. The non-cooperative scheme only uses the V2I links of the LTE system with $40-\mathrm{MHz}$ bandwidth but without the additional $5 \mathrm{MHz}$ out-of-band frequency of another OFDMA-based system. The proposed solution results in a remarkable improvement in SE compared to other schemes. Due to the fact that when the density of vehicles is sparse, the vehicles run faster, leading to bad channel conditions and a lower SE and when the density is too high, the radio resources of $\mathrm{V} 2 \mathrm{~V}$ links are too scarce to all V2V links. Therefore, the SE is the unimodal function of the number of vehicles as shown in Fig. 7.

The average number of cooperative links $N_{V}$ is plotted in Fig. 8 under various numbers of VEs. The value of $N_{V}$ represents the number of VE that adopt the cooperative communication under different levels of traffic density. With the increasing of traffic density, the cooperative links are increasing, which is due to the fact that there are more appropriate VEs which can be served as relay vehicles with

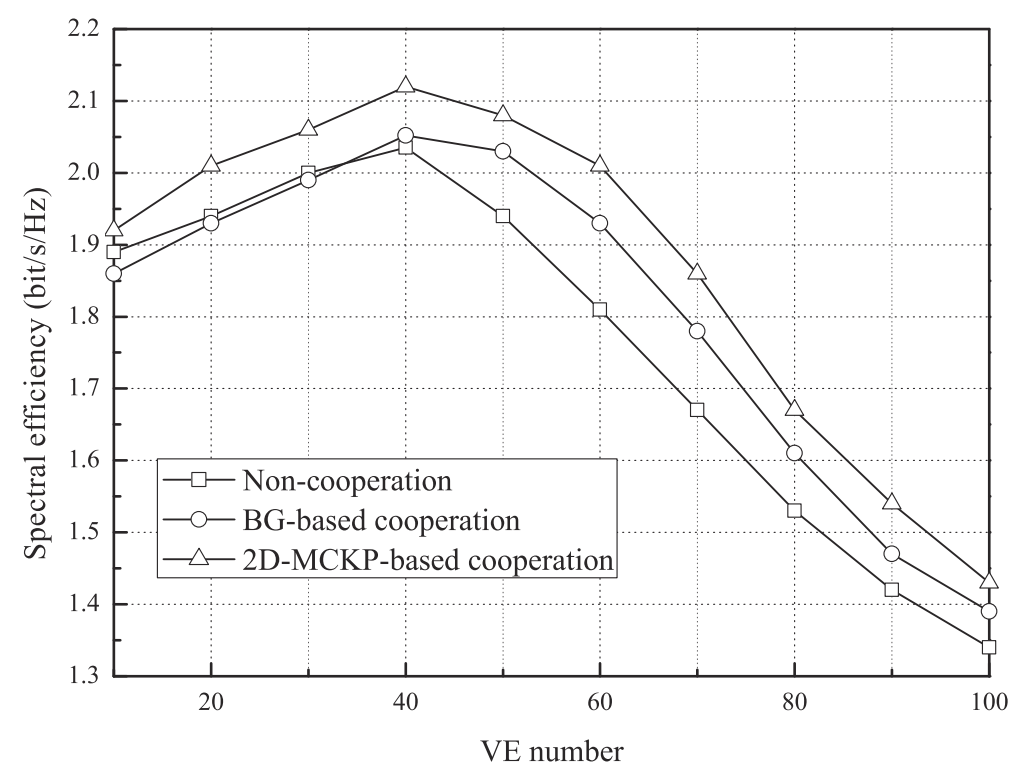

Fig. 7 The comparison of spectral efficiency with different schemes under various VE numbers 


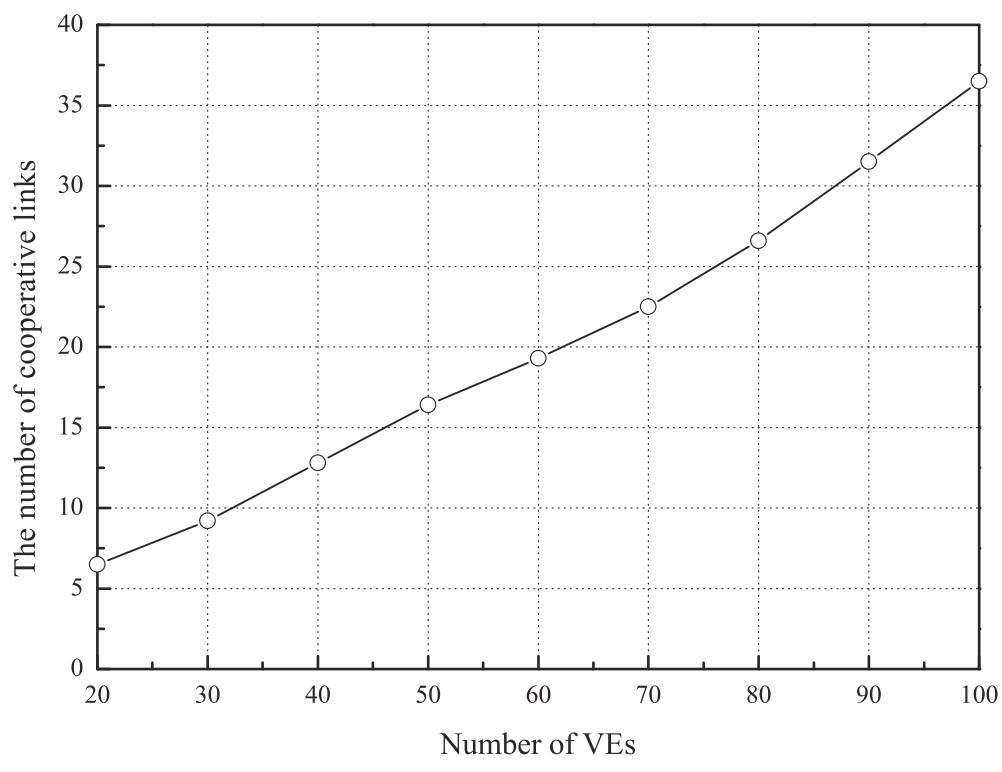

Fig. 8 Average number of cooperative links $N_{V}$ in the 2D-MCKP-based cooperative system under various VE numbers

the increasing of traffic density. As shown in Fig. 8, the number of cooperative vehicles accounts for about $30 \%$ of the number of total vehicles.

\section{Conclusions}

In this paper, the radio link scheduling and resource allocating problem for cooperative vehicular networks are formulated and a joint optimization method based on 2DMCKP algorithm is proposed. In lieu of MSR of the vehicular networks, utility theory is employed to formulate the relationship between user experience and data rate. The optimal link scheduling and resource allocating scheme obtaining the MSU of the vehicular networks is proved to be NP-hard. Therefore, we propose the 2D-MCKP-based scheme, which has an acceptable complexity. To verify the performance of the proposed scheme, we develop a traffic and communication simulator that is based on car-following and random lane-changing models. The simulation results indicate that the performance of the 2D-MCKP scheme is significantly higher than one of the MSR and BG-based schemes. In addition, the 2DMCKP scheme is able to achieve better fairness among VEs than the one of MSR and BG-based schemes and considerably improves the data rate and utility of VEs under poor channel conditions. It is also shown that the 2D-MCKP scheme for cooperative vehicular communications is capable of improving the throughput, as well as the spectral efficiency of vehicular networks, in comparison to the BG-based and non-cooperative scheme.

In future research, we will consider the dynamic process of the arriving data packets and multiple service classifications, for example, infotainment service, traffic safety service, and traffic efficiency service. Dynamic programming theory is employed to optimize the system performance, and end-to-end delay will be considered as a metric to measure the performance for different services.

\section{Competing interests}

The authors declare that they have no competing interests.

\section{Acknowledgements}

This work is funded in part by the National Science Foundation of China (No.61331009), the Fundamental Research Funds for the Central Universities (No. 2014ZD03-02), and the National Key Technology R\&D Program of China under Grant 2014ZX03003011-004.

\section{Author details}

${ }^{1}$ Wireless Signal Processing and Network (WSPN) Lab, Key Lab of Universal Wireless Communications, Ministry of Education, Beijing University of Posts \& Telecommunications, Xitucheng Road, 100876, Beijing, China. ${ }^{2}$ CSSN Research Lab, Department of Informatics, Alexander TEl of Thessaloniki, GR-57400 Thessaloniki, Greece. ${ }^{3}$ Institute for Networked Systems, RWTH Aachen University, D-52072 Aachen, Germany.

Received: 31 August 2014 Accepted: 20 May 2015

Published online: 12 June 2015

\section{References}

1. T Zhou, H Sharif, M Hempel, P Mahasukhon, W Wang, T Ma, A novel adaptive distributed cooperative relaying MAC protocol for vehicular networks. IEEE J. Selected Areas Commun. 29(1), 72-82 (2011). doi:10. 1109/JSAC.2011.110108

2. P Belanovic, D Valerio, A Paier, T Zemen, F Ricciato, CF Mecklenbrauker, On wireless links for vehicle-to-infrastructure communications. Vehicular Technol. IEEE Trans. 59(1), 269-282 (2010). doi:10.1109/TVT.2009.2029119

3. C Sommer, A Schmidt, Y Chen, R German, W Koch, F Dressler, On the feasibility of UMTS-based traffic information systems. Ad Hoc Netw. 8(5), 506-517 (2010)

4. IEEE standard for information technology_local and metropolitan area networks_specific requirements- part 11: wireless LAN medium access 
control (MAC) and physical layer (PHY) specifications amendment 6: wireless access in vehicular environments. IEEE Std 802.11 p-2010, 1-51 (2010). doi:10.1109/IEEESTD.2010.5514475

5. IEEE standard for wireless access in vehicular environments (wave)-networking services. IEEE Std 1609.3-2010 (Revision of IEEE Std 1609.3-2007), 1-144 (2010). doi:10.1109/IEEESTD.2010.5680697

6. IEEE standard for wireless access in vehicular environments (WAVE)multi-channel operation. IEEE Std 1609.4-2010 (Revision of IEEE Std 1609.4-2006), 1-89 (2011). doi:10.1109/IEEESTD.2011.5712769

7. M Seredynski, P Bouvry, in 2011 14th International IEEE Conference on Intelligent Transportation Systems (ITSC). A survey of vehicular-based cooperative traffic information systems, (2011), pp. 163-168. doi:10.1109/ ITSC.2011.6083055

8. K Zheng, F Liu, L Lei, C Lin, Y Jiang, Stochastic performance analysis of a wireless finite-state Markov channel. Wireless Commun. IEEE Trans. 12(2), 782-793 (2013). doi:10.1109/TWC.2012.122212.120223

9. Z Ding, KK Leung, Cross-layer routing using cooperative transmission in vehicular ad-hoc networks. IEEE J. Select. Areas Commun. 29(3), 571-581 (2011). doi:10.1109/JSAC.2011.110307

10. JN Laneman, DNC Tse, GW Wornell, Cooperative diversity in wireless networks: efficient protocols and outage behavior. Inform. Theory, IEEE Trans. 50(12), 3062-3080 (2004). doi:10.1109/TIT.2004.838089

11. I Krikidis, J Thompson, N Goertz, A cross-layer approach for cooperative networks. Vehicular Technol. IEEE Trans. 57(5), 3257-3263 (2008). doi:10. 1109/TVT.2008.915508

12. Q Wang, P Fan, KB Letaief, On the joint V2I and V2V scheduling for cooperative vanets with network coding. Vehicular Technol. IEEE Trans. 61(1), 62-73 (2012). doi:10.1109/TVT.2011.2167249

13. S-Y Pyun, D-H Cho, J-W Son, in IEEE Vehicular Technol. Conf. (VTC Fall). Downlink Resource Allocation Scheme for smart Antenna Based V2V2 Communication System, (2011), pp. 1-6. doi:10.1109/NETECF.2011. 6092854

14. K Zheng, F Liu, Q Zheng, W Xiang, W Wang, A graph-based cooperative scheduling scheme for vehicular networks. IEEE Trans. Vehicular Technol. 62(4), 1450-1458 (2013). doi:10.1109/TVT.2013.2244929

15. R Atat, E Yaacoub, M-S Alouini, F Filali, in IEEE Consumer Communications and Networking Conference (CCNC). Delay Efficient Cooperation in Public Safety Vehicular Networks Using LTE and IEEE 802.11p, (2012), pp. 316-320. doi:10.1109/CCNC.2012.6181109

16. Q Zheng, K Zheng, P Chatzimisios, H Long, F Liu, A novel link allocation method for vehicle-to-vehicle-based relaying networks. Trans. Emerging Telecommun. Technol. (2014). doi:10.1002/ett.2790

17. R Fei, K Yang, X Cheng, in Computer Communications Workshops (INFOCOM WKSHPS), 2011 IEEE Conference On. A Cooperative Social and Vehicular Network and Its Dynamic Bandwidth Allocation Algorithms, (2011), pp. 63-67. doi:10.1109/INFCOMW.2011.5928891

18. E Yaacoub, N Zorba, in Wireless Communications and Mobile Computing Conference (IWCMC), 2013 9th International. Enhanced Connectivity in Vehicular Ad-Hoc Networks via V2V Communications, (2013), pp. 1654-1659. doi:10.1109/IWCMC.2013.6583804

19. G Araniti, C Campolo, M Condoluci, A lera, A Molinaro, LTE for vehicular networking: a survey. IEEE Commun. Mag. 51(5), 148-157 (2013). doi:10. 1109/MCOM.2013.6515060

20. K Zheng, Y Wang, W Wang, M Dohler, J Wang, Energy-efficient wireless in-home: the need for interference-controlled femtocells. Wireless Commun. IEEE. 18(6), 36-44 (2011). doi:10.1109/MWC.2011.6108332

21. JB Kenney, Dedicated short-range communications (DSRC) standards in the United States. Proc. IEEE. 99(7), 1162-1182 (2011). doi:10.1109/JPROC. 2011.2132790

22. V10. 4. 0 3GPP TS 36.211, Technical specification group radio access network (Dec. 2011)

23. MP Wylie-Green, P Wang, in Broadband Communications, Networks and Systems, 2007. BROADNETS 2007. Fourth International Conference On. Utility-Based Cross Layer Optimization for ODDMA Systems Using the $\beta$-Min-Sum Belief Propagation Algorithm, (2007), pp. 459-468. doi:10. 1109/BROADNETS.2007.4550469

24. H Kellerer, U Perschy, Knapsack problems. (Springer

25. A Wegener, H Hellbruck, C Wewetzer, A Lubke, in IEEE GLOBECOM Workshops. Vanet Simulation Environment with Feedback Loop and its Application to Traffic Light Assistance, (2008), pp. 1-7. doi:10.1109/ GLOCOMW.2008.ECP.67
26. S Krauss, Microscopic Modelling of Traffic Flow: Investigation of Collision Free Vehicle Dynamics, (1998). http://e-archive.informatik.uni-koeln.de/319/

27. L Cheng, H Benjamin, SD D., F Bai, P Mudalige, in IEEE Antennas and Propagation Society International Symposium. A Fully Mobile, GPS Enabled, Vehicle-to-Vehicle Measurement Platform for Characterization of the 5.9 GHZ DSRC channel, (2007), pp. 2005-2008. doi:10.1109/APS.2007.4395917

\section{Submit your manuscript to a SpringerOpen ${ }^{\mathcal{O}}$ journal and benefit from:}

- Convenient online submission

- Rigorous peer review

- Immediate publication on acceptance

- Open access: articles freely available online

- High visibility within the field

- Retaining the copyright to your article

Submit your next manuscript at $\boldsymbol{~ s p r i n g e r o p e n . c o m ~}$ 